\title{
Médiévales
}

Langues, Textes, Histoire

54 | printemps 2008

Frères et sœurs

\section{Frères et sœurs ennemis dans la Germanie du $\mathrm{x}^{\mathrm{e}}$ siècle}

Brothers and Sisters at War in Tenth-Century Germany

\section{Laurence Leleu}

\section{OpenEdition}

Journals

Édition électronique

URL : https://journals.openedition.org/medievales/4592

DOI : 10.4000/medievales.4592

ISSN : 1777-5892

\section{Éditeur}

Presses universitaires de Vincennes

\section{Édition imprimée}

Date de publication : 1 juin 2008

Pagination : $35-52$

ISBN : 978-2-84292-217-7

ISSN : 0751-2708

\section{Référence électronique}

Laurence Leleu, «Frères et sœurs ennemis dans la Germanie du xe siècle », Médiévales [En ligne], 54 printemps 2008, mis en ligne le 10 septembre 2010, consulté le 22 avril 2022. URL : http:// journals.openedition.org/medievales/4592; DOI : https://doi.org/10.4000/medievales.4592

Ce document a été généré automatiquement le 22 avril 2022

Tous droits réservés 


\title{
Frères et sœurs ennemis dans la Germanie du $\mathrm{X}^{\mathrm{e}}$ siècle
}

\author{
Brothers and Sisters at War in Tenth-Century Germany
}

Laurence Leleu

1 Le thème des frères ennemis est omniprésent dans la littérature comme dans l'histoire comme le montrent les exemples de Seth et Osiris, Caïn et Abel, ou encore Romulus et Remus : deux ou plusieurs fils, mais un seul trône, un héritage, un honneur, et c'est le conflit. On en a de multiples exemples pour l'ensemble du haut Moyen Âge, qu'il s'agisse des fils et des petits-fils de Clovis, dont les guerres fratricides pimentent le récit de Grégoire de Tours, ou des rivalités entre Charles le Chauve et ses frères Lothaire et Louis le Germanique relatées par Nithard ${ }^{1}$. Il n'en va pas autrement à l'époque ottonienne (919-1024), où la société accorde une importante marge de manœuvre aux femmes dans la gestion de l'héritage ou même dans la politique. Dans ce contexte, les sœurs ne sont pas absentes des conflits «fraternels». Les sources narratives sont certainement celles qui permettent le mieux d'appréhender cette question à travers des cas concrets qui concernent surtout la Saxe, regnum de Germanie le mieux documenté pour la période ; les exemples les plus développés sont évidemment ceux qui concernent la famille royale ottonienne. L'étude de quelques cas devrait permettre de s'interroger sur le rôle du genre, la place dans la fratrie ou encore le remariage d'un des parents après un veuvage ${ }^{2}$ dans les processus conflictuels au sein des fratries.

2 La famille est normalement un espace de paix et de solidarité. Y dominent d'abord les relations entre parents et enfants puis les liens qui unissent frères et sœurs entre eux : «frère » et "sœur » représentent $27,5 \%$ des termes évoquant un lien de parenté consanguine et $23,4 \%$ des termes de parenté toutes catégories confondues dans la Chronique de Thietmar de Mersebourg ${ }^{3}$, avec néanmoins un net avantage à la relation entre frères. Or la notion de fraternité renvoie à un lien horizontal, donc de type égalitaire, unissant entre eux les membres d'une même fratrie ${ }^{4}$. Dans les sources, particulièrement chez Thietmar de Mersebourg, le vocabulaire employé pour définir et décrire ce lien insiste sur sa forte charge affective. L'auteur lui-même, membre d'une importante fratrie ${ }^{5}$, évoque à de nombreuses reprises ses frères, toujours dans des 
termes affectueux : par exemple, il s'adresse à son jeune frère Siegfried «lié et cher à [lui] par l'amour de la douce fraternité ${ }^{\prime} »$. Cette solidarité fraternelle, conçue et présentée comme la norme ${ }^{7}$, s'explique en grande partie par le maintien des droits des cadets et des filles à l'héritage en Germanie ottonienne. En Saxe, dans les fratries nombreuses, le plus souvent deux frères au moins restent dans le monde ${ }^{8}$, et dans ce cas, soit ils se partagent la puissance paternelle, soit l'héritage est maintenu en indivision et la puissance est exercée conjointement par les frères. Dans tous les cas, ils sont amenés à être solidaires. Ce type d'arrangements, destiné à éviter les jalousies et à générer une coopération, constitue le terreau de liens qui sont la plupart du temps particulièrement privilégiés et forts : les sources de l'époque proposent de nombreux exemples de frères et/ou de sœurs qui se soutiennent et s'entraident. Le plus fameux est sans doute celui de Brunon de Cologne ${ }^{9}$, soutien indéfectible de son frère Otton $\mathrm{I}^{\text {er10 }}$, chancelier à partir de 941, archichapelain à partir de 951, archidux ${ }^{11}$ de Lotharingie après la révolte de Liudolf et du duc Conrad le Rouge en 953. On le voit aussi en 956 réconcilier son neveu Liudolf avec son père ${ }^{12}$, Otton I ${ }^{\text {er }}$. Sa mort en 965 laisse l'empereur affligé aux dires de Thietmar ${ }^{13}$.

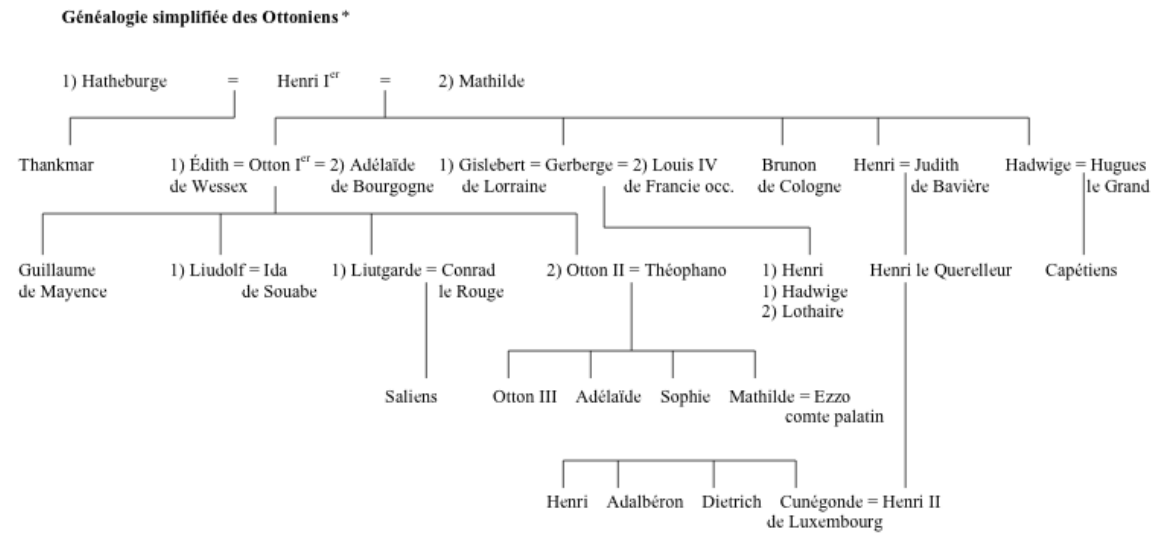

Un autre exemple est tout à fait révélateur de la solidarité entre frères et sœurs cette fois : celui de l'attitude de la mère de Thietmar quand ses frères sont faits prisonniers par les Vikings. L'évêque raconte en effet l'expédition de ses trois oncles maternels, Henri, Udo et Siegfried, contre des pirates danois en 994. Udo meurt dans la bataille et les deux autres, battus, sont gardés captifs. Voici la réaction de leur sœur Cunégonde à l'annonce de cette nouvelle: "Ma mère fut bouleversée par une telle douleur qu'elle donna tout ce qu'elle possédait et avait pu acquérir par tous les moyens possibles pour la libération de ses frères. » En plus de la rançon versée, les pirates mettent en place un système d'échange d'otages : Henri est remplacé par son fils Siegfried; le second frère, lui aussi dénommé Siegfried, n'ayant pas de descendant, fait demander à sa sœur Cunégonde d'envoyer l'un des siens pour prendre sa place : après le refus du premier, c'est Thietmar qui se dévoue et se rend auprès des Danois, sous l'habit laïc, afin de faire libérer son oncle, entre-temps évadé ${ }^{14}$. On voit que pour sa mère, l'attachement à ses 
frères est aussi puissant que celui qu'elle porte à ses propres enfants, puisqu'elle accepte de risquer la vie de l'un de ses fils, ecclésiastique néanmoins, pour sauver Siegfried des griffes de l'ennemi. Plus globalement, en Saxe particulièrement, les sœurs jouent un rôle fondamental dans l'entretien de la mémoire de leurs frères dans le cadre des monastères féminins ${ }^{15}$.

Malgré ces beaux exemples, il existe aussi une violence extrême car intrafamiliale, qui brise la solidarité et la paix normalement préservées au sein de la famille, et qui remet en cause la concordia et son corollaire, l'ordre. Les sources condamnent unanimement ces conflits familiaux: issus d'un comportement perçu comme anormal, ils transgressent l'ordre établi sur lequel se fonde la paix sociale et installent le désordre. Certaines sources vont même jusqu'à les passer sous silence pour soutenir l'idéologie de concordia du régime ottonien ${ }^{16}$. Les origines de ces conflits se situent le plus souvent dans le sentiment d'injustice et de jalousie ressenti par un des membres de la famille qui considère ses intérêts comme lésés. La famille apparaît donc comme une réalité sociale complexe, comme le siège d'intérêts parfois divergents, à l'origine de conflits plus nombreux et quelquefois même beaucoup plus violents qu'on ne pourrait s'y attendre. Ce sont ces conflits entre frères et sœurs que je vais analyser ici, en distinguant trois types de cas qui seront étudiés en fonction de leur gravité et de leur durée, du simple désaccord à la faide familiale, en passant par le conflit ouvert et généralement armé.

Conflits entre frère et sœur, conflits entre beaux-frères

5 Le premier exemple est celui du conflit entre Gerberge et ses frères. Fille du roi Henri $\mathrm{I}^{\mathrm{er}}$ de Germanie et sœur d'Otton I ${ }^{\mathrm{er}}$, elle présente un parcours intéressant en tant que figure ambivalente de la soror regina comme la qualifient les sources germaniques de l'époque ${ }^{17}$. Son premier époux, Gislebert de Lotharingie ${ }^{18}$, choisit en 939 de suivre son beau-frère Henri de Bavière dans sa révolte contre Otton $\mathrm{I}^{\mathrm{er}}$, ce dont nous reparlerons. Une seule source évoque l'attitude de Gerberge dans cette affaire qui oppose son mari et un de ses frères d'un côté à un autre de ses frères de l'autre : c'est la Translatio sancti Servatii datant $\mathrm{du} \mathrm{XI} \mathrm{I}^{\mathrm{e}}$ siècle ${ }^{19}$ qui présente la princesse de façon plutôt négative, comme l'instigatrice de la rébellion de son mari contre le roi de Germanie. En raison du silence $\mathrm{du}$ reste de la documentation, il est difficile d'infirmer ou de confirmer cet avis, mais il semble plutôt logique de penser que Gerberge a alors agi conformément à ce que l'on attendait d'une épouse dévouée et qu'elle a donc soutenu son mari et les intérêts de ses enfants, même contre son propre frère, dans ce conflit politique intrafamilial. Après la mort de Gislebert ${ }^{20}$, elle s'unit au roi de Francie occidentale Louis IV d'Outremer ${ }^{21}$ contre le gré de son frère ${ }^{22}$, qui n'a même pas été consulté dans cette affaire : c'est d'ailleurs là tout le problème, car le nouveau roi, depuis la mort de leur père Henri, se considère comme investi de la potestas paternelle auprès de sa sœur, avec autorité sur elle, et notamment le droit de la marier en fonction de ses intérêts et des stratégies familiales. La brouille entre le frère et la sœur trouve ici son origine simplement dans la trop grande liberté que Gerberge, jeune veuve attractive par ses droits sur la Lotharingie ${ }^{23}$, s'est octroyée dans la décision d'une affaire d'ordinaire considérée comme celle de la famille tout entière, et qui revenait la plupart du temps au chef de celle-ci ${ }^{24}$. Pourtant, Otton tire bien vite avantage de ce mariage qui lui assure une position d'arbitre en Francie occidentale, entre les familles carolingienne et robertienne, puisque son autre sœur, Hadwige, a épousé le duc des Francs Hugues le Grand en $937^{25}$. Par la suite, les visites de la reine Gerberge à son frère Otton I ${ }^{\text {er }}$ montrent leur bonne entente et l'entraide des souverains de Francie et de Germanie ${ }^{26}$. 
Après la mort de Louis, la reine de Francie occidentale reçoit la protection de ses frères, Otton et Brunon, et ce dernier, archevêque de Cologne, joue même pour son neveu Lothaire un rôle proche de celui de régent. Entre les frères et la sœur une solidarité retrouvée et une collaboration poussée permettent d'asseoir l'hégémonie ottonienne en Occident.

6 Le cas de Gerberge, deux fois en conflit ou plutôt en opposition avec son frère Otton, permet de souligner un certain nombre d'aspects importants des relations spécifiques des frères avec leurs sœurs à l'époque ottonienne. L'idéal est bien sûr la bonne entente au sein de la fratrie, soulignée par l'insistance des sources sur les liens de parenté (soror regina) et d'affection, mais celle-ci peut connaître des hauts et des bas, particulièrement si la sœur est mariée ou veuve. En effet, une fois mariée, la relation adelphique s'efface devant la relation conjugale et les devoirs de la mère. Les liens fraternels sont apparemment moins contraignants que ceux du mariage et surtout de la maternité... Il apparaît clairement que, même si l'alliance a alors pour vocation principale l'établissement ou la confirmation de la paix entre deux familles, les conflits ne sont pas pour autant exclus entre beaux-frères. Il semble même que les désaccords concernent bien plus ceux-ci, car les frères et sœurs entrent rarement en concurrence dans la mesure où ils n'ont pas la même trajectoire sociale ${ }^{27}$, à l'inverse des beauxfrères. C'est ce que montre l'exemple des frères de la reine Cunégonde révoltés contre le roi Henri II : celui-ci, peu après son avènement en 1002, confie son ancien duché de Bavière à Henri, frère de son épouse, et resserre d'autant ses liens avec lui ${ }^{28}$. Mais cela ne suffit pas à empêcher la révolte : en 1009, Henri rejoint celle fomentée par ses frères, les évêques Adalbéron de Trèves et Dietrich de Metz, déçus dans leurs ambitions, contre leur beau-frère; la sanction est immédiate : déposition et excommunication ${ }^{29}$. Une fois la réconciliation conclue, en 1017 seulement $^{30}$, Cunégonde peut procéder à la réinstallation de son frère en signe de pardon ${ }^{31}$. Cela confirme un certain nombre de constantes anthropologiques: la solidarité fraternelle contre un même ennemi, les difficiles relations entre beaux-frères, le rôle de médiateur de la sœur et épouse entre les deux camps : elle cherche à favoriser ses frères, mais sans pour autant prendre leur parti une fois qu'ils entrent dans la dissidence. Elle se range alors du côté de son époux tout en cherchant vraisemblablement à calmer les esprits et à aider à la réconciliation. Elle joue ainsi pleinement son rôle de lien auprès de ses deux familles.

Les frères ennemis : violence et réconciliation

7 Une fratrie n'est pas toujours composée d'individus nés des deux mêmes parents. Au Haut Moyen Âge, les conditions sont même réunies pour favoriser la constitution de familles recomposées: l'espérance de vie est faible et les causes de mortalité nombreuses car les décès en couches pour les femmes et les conséquences des guerres pour les hommes causent de nombreux veuvages, et donc des remariages assez fréquents. L'Église tolère cette pratique même si elle ne l'encourage pas. Au contraire, elle diffuse une discipline matrimoniale qui passe par l'interdiction de la répudiation, pratique dont on trouve peu trace dans l'espace germanique au-delà du début du $\mathrm{x}^{\mathrm{e}}$ siècle ${ }^{32}$.

8 Le fait d'être le fils de l'épouse légitime a en effet son importance à une époque où l'Église tend à imposer son modèle marital comme l'illustre l'exemple de Thankmar. Il est le fils aîné du roi Henri $\mathrm{I}^{\mathrm{er}}$ mais celui-ci a répudié sa mère Hatheburge ${ }^{33}$, prétextant de l'irrégularité de cette union ${ }^{34}$, pour épouser Mathilde, ce qui a affaibli la position de Thankmar dans la famille. Pourtant, sa révolte en 938 contre son demi-frère Otton 
récemment sacré roi, ne semble pas motivée, comme on pourrait le croire, par une quelconque revendication d'un droit au trône. Widukind et Thietmar s'accordent sur le fait que le jeune homme réclame avant tout l'héritage de sa mère ${ }^{35}$. En effet, Henri, ayant répudié sa première épouse, s'est bien gardé de lui restituer les biens qu'elle avait reçus en héritage de son père Erwin, senior de Mersebourg. De plus, le jeune homme revendique la legatio de son cousin Siegfried, comte de Mersebourg, attribuée par le roi au margrave Gero ${ }^{36}$. D'où un soulèvement qui conteste son éviction flagrante des honneurs ; le conflit ne se résout que par sa mort accidentelle dans l'église d'Eresbourg, alors qu'il s'apprêtait à se soumettre au pouvoir et à la décision de son frère, Otton $\mathrm{I}^{\text {er37 }}$. Il s'agit donc ici d'une simple contestation d'héritage, dans une situation particulière de cohabitation de frères issus de lits différents.

9 Le cas de Liudolf est plus équivoque. Il est issu du mariage d'Otton $\mathrm{I}^{\mathrm{er}}$ avec Edith, une princesse anglo-saxonne ${ }^{38}$. Sa révolte en 953 fait suite à la mort de sa mère et surtout au remariage de son père. Mais elle est surtout due au fait qu'Henri de Bavière, jeune frère $\mathrm{du}$ roi, voit grandir son influence à la cour et qu'il adopte en public une attitude méprisante envers son neveu ${ }^{39}$. Le soulèvement de Liudolf apparaît donc bien moins tourné contre son père que contre son oncle ${ }^{40}$ qui semble contester sa désignation comme héritier du trône. En fait, il est probable que le remariage d'Otton avec la jeune Adélaïde porte atteinte à la position de fils unique de Liudolf et lui fasse redouter la très plausible naissance d'un demi-frère mieux entouré et soutenu, qui pourrait donc remettre en cause sa désignation comme successeur au trône. On pourrait donc aussi y voir un soulèvement contre un demi-frère à venir et déjà perçu comme menaçant.

10 La situation n'est pas moins compliquée entre frères nés des deux mêmes parents. Revenons à Henri de Bavière, «premier dans le royaume après le roi $»^{41}$ : entre 938 et 941, il apparaît comme une sorte de révolté récidiviste qui n'accepte pas les modalités de la succession royale. Hrotsvitha de Gandersheim souligne à propos de la succession d'Henri $\mathrm{I}^{\mathrm{er}}$ : «La Trinité avait donné au roi trois fils par une disposition qui rendit la gens heureuse, afin que les hommes méchants n'arrachent pas le regnum après la mort d'Henri, le roi vénérable. [...] Mais on a disposé pour eux des honneurs dissemblables parce que deux avaient été soumis à l'un qui régnait ${ }^{42}$." $A u \mathrm{X}^{\mathrm{e}}$ siècle, les règles de succession évoluent en faveur de l'indivisibilité du royaume, ce qui crée des conflits à l'intérieur de la famille régnante pourvue de plusieurs fils adultes et restés laïcs. En effet, le système favorise une primogéniture modulable ${ }^{43}$ qui désavantage les cadets. Le problème se rencontre dès la mort $\mathrm{d}^{\prime} H e n r i \mathrm{I}^{\mathrm{er}}$ : bien qu'Otton ait été désigné dans les règles lors de la réunion d'Erfurt de $936^{44}$, où des compensations sont prévues pour les autres fils, le nouveau souverain rencontre très vite l'opposition de son cadet Henri "brûlant du désir de régner", comme l'écrit à de nombreuses reprises Widukind ${ }^{45}$. Celui-ci, apparemment soutenu par sa mère la reine Mathilde ${ }^{46}$, fonde son argumentation, comme le dit la Vita Mathildis reginae posterior, sur le fait qu'il est le premier natus in aula regali ${ }^{47}$, c'est-à-dire le premier né après l'accession de son père à la royauté, argument reprenant la tradition byzantine des Porphyrogénètes. Dans ce contexte, deux absences se font remarquer lors du sacre royal de 936 à Aix-la-Chapelle : celle de Mathilde, restée en Saxe, déçue qu'Henri, son fils préféré, n'ait pas été choisi pour succéder à son père $e^{48}$, et celle d'Henri lui-même ${ }^{49}$. Afin d'éviter toute contestation pendant la cérémonie, celui-ci a été éloigné du lieu du sacre sous la garde du comte palatin de Saxe Siegfried. Le jeune frère d'Otton montre d'ailleurs très tôt ses prétentions au trône et ses tentatives de prise de pouvoir constituent les premières embûches rencontrées par le jeune roi. Le premier épisode de cette lutte qui deviendra 
attentat fratricide se situe en 938-939 et nous est rapporté par Widukind : " Henri était à cette époque encore un très jeune homme ${ }^{50}$ au caractère bouillant ; et, égaré par une trop grande envie de régner, il est corrompu par un pacte criminel, selon lequel, par une conjuration contre le roi son seigneur et frère, il ceindrait la couronne royale, si cela était possible ${ }^{51}$. » Henri complote à deux reprises et, découvert, reçoit à chaque fois le pardon de son frère ${ }^{52}$. Enfin, dernier essai, la tentative d'assassinat d'Otton par les partisans de son frère à Pâques en 941 à Quedlinbourg et dont l'échec amène Henri à quitter le royaume ${ }^{53}$. Le problème soulevé par Henri le Jeune s'est apaisé progressivement à partir de la décision d'Otton $\mathrm{I}^{\mathrm{er}}$ de lui attribuer le regnum de Bavière en le poussant dès 938 à s'unir à Judith, fille du duc Arnulf ${ }^{54}$. La mort de ce dernier en 945 permet ainsi à Henri de créer une nouvelle dynastie bavaroise et d'obtenir une fraction de ce pouvoir si recherché par lui.

Dans ces différents passages, le motif de la révolte et des complots apparait clairement : il s'agit pour Henri de contester la décision de son père faisant d'Otton son seul successeur, en proclamant sa volonté de régner avec lui peut-être au départ, sur le modèle franc de la conception patrimoniale du royaume, puis très vite à la place même de son frère. Quoi qu'il en soit, il semble que l'application de cette règle de succession marque pour la famille ottonienne le passage à une nouvelle structure d'organisation, celle de la lignée, caractérisée notamment par l'instauration de la primogéniture et donc de la prééminence de l'aîné. Les tentatives d'Henri montrent la résistance d'un membre de la famille à cette évolution, afin de préserver entre les héritiers des droits strictement égaux. Ici, la violence naît du refus d'un nouveau type d'ordre familial, qui fait des cadets, non les grands lésés, mais ceux qui doivent se contenter de la seconde place. L'appartenance de l'usurpateur en herbe à la famille royale explique sans doute la clémence extraordinaire d'Otton à son égard: jamais Henri n'est puni pour ses attaques répétées contre le pouvoir de son aîné; Otton reconnaitrait-il la valeur des désirs de son frère ? Pour restaurer l'ordre, la violence doit néanmoins être suivie du pardon, de la clementia qui est le propre des souverains. Les rituels sont là pour réaffirmer la hiérarchie et la supériorité $d u$ roi $^{55}$. Le fauteur de trouble, le mauvais frère, battu ou repentant, se rend finalement de son plein gré auprès du souverain pour lui demander pardon. Jean-Marie Moeglin explique cela par l'assurance pour le coupable de ne pas être puni de façon trop sévère ${ }^{56}$ : faute avouée est à demi pardonnée. Ainsi est renoué le lien rompu par le conflit grâce à un rituel bien précis qui permet le retour à l'équilibre social et à la paix familiale. Un passage particulièrement frappant et détaillé nous est fourni par Hrotsvitha de Gandersheim au sujet de l'amende honorable du belliqueux Henri de Bavière, intervenue en 941, après plusieurs années d'insubordination et de révolte vis-à-vis de l'autorité de son frère Otton $\mathrm{I}^{\mathrm{er}}{ }^{57}$ : elle décrit tout d'abord les remords qui assaillent le frère du roi, puis, « vaincu par la force de son amour, il repoussa de son cœur la crainte de la punition et, arrivant dans le secret de la nuit, il vint dans la ville royale, dans laquelle le pieux roi commençait à célébrer la Naissance du Roi Éternel avec la déférence appropriée ». Revêtu de vêtements simples et pieds nus, il se prosterna devant l'autel de l'église, «souhaitant ainsi, par l'élan de son cœur repentant, recevoir le doux cadeau du pardon. Alors, vaincu par une généreuse tendresse, [...] en l'honneur d'un tel jour porteur de paix, le roi compatit, déplorant les fautes de son frère qui les reconnaissait, et avec affection il lui accorda sa grâce ainsi que le présent apprécié de son total pardon.» Puis la chanoinesse précise : «Et après cela, il n'y eut plus d'autre discorde entre eux, leurs âmes étant liées par une alliance fraternelle. $\aleph^{58}$ Même si Hrotsvitha tente de nous le faire croire, ce geste n'a 
absolument rien de spontané, puisqu'il s'agit justement d'un rituel ; d'ailleurs, le choix d'Henri du soir de Noël n'a bien entendu rien d'innocent comme le suggère le passage même. Cette mise en scène accomplie, les conditions sont réalisées pour l'organisation d'une fête, avec pour cœur le fameux convivium ou festin qui scellera la fin des hostilités ${ }^{59}$. La crise passée, l'ordre est restauré, preuve de la légitimité de cet ordre ${ }^{60}$.

Néanmoins, dans des cas extrêmes, le conflit entre frères et sœurs peut déboucher sur de véritables faides familiales, comme dans le cas des Billung et des Hamaland.

La faide familiale

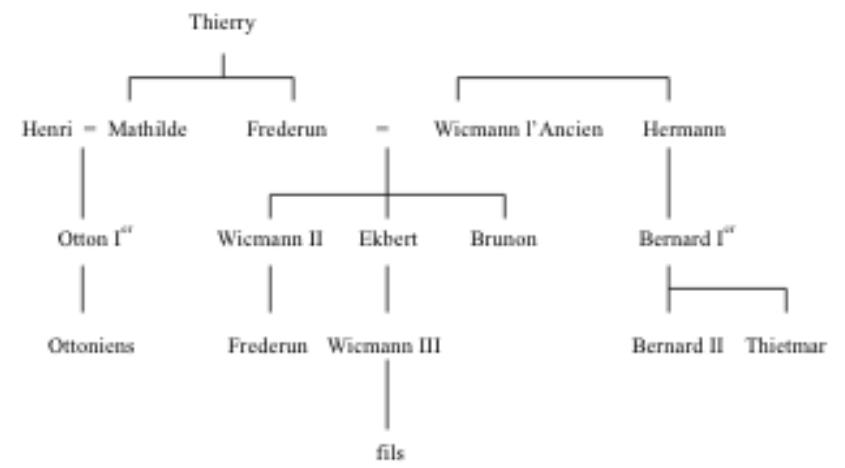

13 La faide des Billung, qui scinde pendant de nombreuses années cette famille, trouve son origine dans une intervention d'Otton $\mathrm{I}^{\mathrm{er}}$ pour favoriser la branche cadette, celle d'Hermann, en donnant à celui-ci la charge du duché de Saxe en 936, ce qui provoque l'invidia de son frère aîné Wicmann ${ }^{61}$. La jalousie déclenchée par cette offensio débouche sur une faide qui oppose pendant de longues années les deux branches de la famille issues des deux frères ${ }^{62}$. En violent conflit, elles mènent longtemps une politique concurrente, notamment pour le contrôle des monastères ${ }^{63}$. Par cette décision lourde de conséquences, le roi est en effet intervenu dans l'organisation familiale et a ainsi procédé à une véritable restructuration de celle-ci : c'est le cadet qui est préféré à l'aîné, ce qui provoque la haine et la volonté de vengeance de celui-ci, d'autant plus qu'il est l'époux de Frederun, sœur de la reine Mathilde, et donc allié direct de la famille ottonienne. On retrouve ici un conflit né d'un sentiment d'injustice dû à des intérêts perçus comme lésés, mais qui cette fois tarde à trouver une solution compensatoire satisfaisante. En effet, le « déclassement » de Wicmann face à son cadet provoque celui de tous ses descendants. Les sources narratives ne rapportent pas d'autres indications au sujet de Wicmann l'Ancien, si ce n'est une brève participation à la révolte de Thankmar en $938^{64}$, mais en revanche elles s'attardent sur la révolte de ses fils Wicmann II et Ekbert contre leur oncle, qui sont même prêts à demander l'aide des Slaves contre lui ${ }^{65}$, et qui choisissent systématiquement des camps opposés dans toutes les grandes affaires agitant la Saxe ${ }^{66}$. Les manifestations d'animosité se poursuivent même après la mort d'Hermann en 973 : Brunon, évêque de Verden et troisième fils de Wicmann, refuse que son oncle, qu'il avait excommunié de son vivant, soit enterré à Saint-Michel de Lunebourg, cloître pourtant fondé par le duc défunt ${ }^{67}$. Ce n'est qu'avec le recentrage des possessions de la branche de Wicmann en Saxe occidentale que la réconciliation peut avoir lieu : en 1016, Bernard II s'institue tuteur des fils de Wicmann III ${ }^{68}$ et fait ainsi reconnaître à toute la parentèle la supériorité de la branche ducale, positionnée en Saxe du Nord. 

Liutgarde. Adèle est un personnage célèbre par la « légende noire » qu'elle a suscitée à plus ou moins juste titre ${ }^{69}$ : impliquée dans une faide contre Wicmann Billung dans la région du Bas-Rhin, elle nous intéresse ici par son refus des aliénations successives du patrimoine familial réalisées pro remedio animae par son père Wicmann, comte du Hamaland, et sa sœur Liutgarde, au profit du monastère familial d'Elten ${ }^{70}$. Il est intéressant de constater combien Alpert de Metz, dont le De Diversitate temporum constitue la principale source sur cette affaire ${ }^{71}$, oppose systématiquement les deux sœurs. Adèle apparaît vraiment comme une femme mauvaise et perverse, qui manipule son mari pour servir ses intérêts, une furie, l'exact contraire de la douce et vertueuse abbesse d'Elten Liutgarde ${ }^{72}$. Le conflit foncier entre les deux sœurs se double de la vive opposition de Liutgarde au remariage de la comtesse avec un homme de naissance obscure, ce qui "dégénère" la famille selon Alpert ${ }^{73}$. À la mort de l'abbesse, empoisonnée, vers 996, Adèle est soupçonnée, d'autant qu'elle tente alors de récupérer les terres convoitées. Elle mène une lutte d'une dizaine d'années pour récupérer ce qu'elle considère comme ses biens, car ceux-ci ont été donnés sans son consentement ${ }^{74}$. Bref, une simple affaire de patrimoine contesté qui tourne à la tragédie, et révèle une femme qu'on peut qualifier de « gardienne inflexible du patrimoine familial $»^{75}$.

harmonieuses et même affectueuses, les tensions, voire de graves conflits ont bien existé à l'époque ottonienne, comme les sources évoquées ici même le montrent, ce qui brise quelque peu l'image trop idyllique que l'on peut avoir des relations familiales, et notamment adelphiques, théoriquement fondées au Haut Moyen Âge sur la solidarité et l'entraide. Ces conflits naissent généralement des mêmes causes: un sentiment d'injustice fort, donc des intérêts perçus comme lésés, dans le cadre le plus souvent d'une revendication d'héritage ou d'honneur. Ces brouilles concernent surtout deux frères (aîné/cadet) ou demi-frères laïcs, alors que les relations frère/sœur et frères laïc/ecclésiastique sont plus paisibles, ce qui s'explique par l'absence de compétition entre eux pour les honneurs, en raison de leurs destins sociaux différents liés au genre ou au type de carrière poursuivi. Mais il est frappant de constater que les cadets n'utilisent pas souvent cette possibilité dans les exemples évoqués ici, preuve que leur statut ne s'est pas réellement dégradé quand s'amorce en Germanie la structuration de la famille autour de lignées. Il y a apparemment ici une différence nette avec ce qui se passe à l'Ouest, où la situation des cadets se détériore bien plus avec le passage à la structure lignagère ${ }^{76}$.

16 Les sources présentent toujours le conflit adelphique comme anormal, condamnable, contre nature, comme la conséquence d'une corruption. Les blâmes sont particulèrement virulents contre ceux qui en sont à l'origine : on leur reproche leur caractère peu équilibré : leur immaturité et leur impatience, leur oubli de l'amour fraternel et leur ingratitude (Henri), leur nature mauvaise et méchante (Adèle), leur jalousie (Wicmann)... De telles constructions masquent peut-être des possibilités d'actions individuelles s'exerçant contre les intérêts du groupe. Quoi qu'il en soit, celui qui rompt l'unité fraternelle est coupable de ce qui s'apparente à un crime ou à un pêché lié à celui, mortel, d'envie, et il menace gravement l'avenir et l'intérêt familiaux par son comportement scandaleux. Il est d'ailleurs frappant que Thietmar de Mersebourg explique le conflit entre Otton $\mathrm{I}^{\mathrm{er}}$ et son cadet Henri par l'intervention du Diable dans la conception de ce dernier un soir d'ivresse; le Malin prophétise même : 
«la Discorde ne le quittera jamais ni sa descendance. Ils ne connaîtront jamais la paix ${ }^{77}$. " À noter enfin qu'à ma connaissance, dans un conflit, les torts ne sont jamais présentés comme partagés: vision un peu manichéenne qui confronte le bon et le mauvais (Liutgarde/Adèle), le sage et l'irréfléchi (Otton/Henri), bref, encore une fois l'ordre et le désordre.

Néanmoins, les sources étudiées ne présentent pas de cas de fratricide concrétisé (car on a vu que les partisans d'Henri de Bavière préparent en 941 l'assassinat d'Otton I ${ }^{\text {er }}$ mais qu'ils échouent) en Germanie même, ce qui permet de nuancer la gravité des conflits adelphiques à l'époque ottonienne. Le seul mentionné concerne le monde slave : le bon duc chrétien des Bohêmiens Wenceslas est tué par son méchant frère le païen Boleslas ${ }^{78}$. Cet exemple est révélateur du regard négatif et dépréciatif porté par Thietmar de Mersebourg sur les Slaves, qui décidément ne respectent rien et apparaissent presque comme des hommes dénaturés, à la limite de la sauvagerie, sans aucun sens de la famille ${ }^{79}$.

\section{NOTES}

1..GRÉGOIRE DE TOURS, Decem libri historiarum, B. KRUSCH éd., MGH., S.S. rer. Merov., I, 1, Hanovre, 1937, R. LATOUCHE trad., Histoire des Francs, 2 vol., Paris, 1963-1965 ; NITHARD, Histoire des fils de Louis le Pieux, P. LAUER éd. et trad., (Les Classiques de l'Histoire de France au Moyen Âge 7), Paris, 1926.

2..Sur tous ces aspects, D. LETT, Histoire des frères et sœeurs, Paris, 2004.

3..THIETMAR DE MERSEBOURG, Chronicon, R. HOLTZMANN éd., MGH., SS rer. Germ. Nova Series IX, HanovreLeipzig, 1935, éd. Berlin 1955. Cette édition (que j'utilise) est reprise dans Thietmar von Merseburg. Chronik, W. TRILLMICH trad., Ausgewählte Quellen zur deutschen Geschichte des Mittelalters IX, Berlin, 1977, nouvelle éd. 2002. Ces pourcentages sont élevés au regard des résultats obtenus par l'étude d'Isabelle RÉAL sur les chroniques mérovingiennes dans Vies de saints, vie de famille. Représentation et système de parenté dans le Royaume mérovingien (481-751) d'après les sources hagiographiques, Turnhaut, Hagiologia 2, 2001, p. 117 : 15\% des termes de parenté concernent les liens de fraternité (étudiés p. 476-492).

4..R. LE JAN, Famille et pouvoir dans le monde franc (VII'-Xe siècle). Essai d'anthropologie sociale, Paris, 1995, p. 79.

5..Il a quatre frères (Henri, Frédéric, Siegfried et Brun), un frater ex patre (Willigis, expression que l'on trouve en VI, 47, p. 332) tous mentionnés ; on lui connaît par ailleurs une sœur, Oda, qui n'apparaît pas dans la Chronique. Voir H. LIPPELT, Thietmar von Merseburg. Reichsbischof und Chronist, Cologne-Vienne, 1973.

6..THIETMAR, I, Prologue, p. 3 : « Coniuctum dulci fraterni iuris amore/ Et mihi dilectum supplex te rogo Sigefridum... » Il déplore seulement en VI, 44, p. 328 que ses frères ne l'aient pas soutenu pour obtenir la totalité de l'abbaye familiale à moindre coût, face à leur oncle paternel Liuthar qui réclamait une forte somme : c'est la preuve de la conception de la relation fraternelle comme devoir réciproque d'entraide. Celuici paraît ici peu efficient, sans doute car Henri et Frédéric ont déjà cédé leur part à Thietmar et doivent considérer en avoir fait assez en sa faveur dans cette affaire.

7..Les sources normatives insistent beaucoup sur l'entente, l'affection, le respect qui doivent lier les parents : par exemple, BURCHARD DE WORMS, Decretum libri XX. Ergänzter Neudruck der Editio Princeps Köln 1548, G. FRANSEN et T. KÖLZER éd., Aalen, 1992, XVI, 28 (sans pagination). Ces mêmes sources condamnent très 
violemment les différends familiaux, particulièrement entre parents et enfants et entre frères et sœurs : celui qui hait son frère est considéré comme homicide. Sur ce point par exemple RABAN MAUR, Lettre 56, E. DÜMMLER éd., MGH., Epistolae Karolini Aevi, III, Berlin, 1899, p. 509-514, ici p. 511 ; Capitula Troleiana, R. POKORNY éd., M.G.H., Capitula episcoporum, III, Hanovre, 1995, p. 141-146, ici c. 6, p. 145 : « Ut nullus discordiam ferat cum proximo suo, sed unum cor et una anima scilicet fratribus in Christo consotient, quoniam, qui odit fratrem suum, homicida est », BURCHARD DE WORMS, I, 131.

8..R. SCHÖLKOPF, Die sächsischen Grafen (919-1024), Göttingen, Studien und Vorarbeiten zum historischen Atlas Niedersachsen 22, 1957, p. 18. R. LE JAN, « De la France du Nord à l'Empire : réflexions sur les structures de parenté au tournant de l'an mil », dans P. BONNASSIE et P. TOUBERT éd., Hommes et sociétés dans l'Europe de l'an mil, Toulouse, 2004, p. 163-184, donne des exemples du même phénomène dans le reste de la Germanie. 9..Généalogie simplifiée de la famille ottonienne en annexe, Tableau n ${ }^{\circ} 2$. Sur ce personnage de Brunon, voir F. LOTTER, « Das Bild Brunos I. von Köln in der Vita des Ruotgers », Jahrbuch des kölnischen Geschichtsvereins, $\mathrm{n}^{\circ} 40,1966$, p. 20-40 et plus récemment P. CORBET, Les Saints ottoniens. Sainteté dynastique, sainteté royale et sainteté féminine autour de l'an mil, Sigmaringer, (Beihefte der Francia), 1986, p. 50-57 et p. 74-79.

10..Je ne tiens pas compte ici de l'épisode, très contestable, relaté par le seul Thietmar de Mersebourg, d'une hypothétique implication de Brunon dans un complot visant à donner la couronne à Hugues le Grand duc des Francs occidentaux ou, plus vraisemblablement, à Conrad le Rouge de Lotharingie : THIETMAR, II, 23, p. 66. L'intervention divine permet au projet d'avorter et aux deux frères de se réconcilier. Si l'on est à peu près sûr du caractère fictif de ce récit, sa signification reste problématique : exprime-t-il une volonté de restaurer un royaume de Lotharingie indépendant ? Est-il le reflet de la volonté de l'archevêque de Cologne de retrouver la prééminence dans le rituel du sacre face à celui de Mayence ?

11..RUOTGER, Vita Brunonis archiepiscopi Coloniensis, G. H. PERTZ éd., MGH., SS IV, Hanovre, 1841, éd. 1968., p. 254-275, ici c. 20, p. 261.

12..Ibid., c. 36, p. 268-269.

13..THIETMAR, II, 23, p. 68.

14..Ibid., IV, 23-25, p. 158-162.

15..Par exemple, ibid., III, 10, p. 108 : fondation du monastère d'Alsleben à la mémoire du comte Gero par sa sœur Tetta et son épouse Adela.

16..P. BUC, Dangereux rituel. De l'histoire médiévale aux sciences sociales, Paris, 2003, traduction de l'ouvrage en anglais de 2001, chapitre 1 « Comment mettre en texte l'hégémonie ottonienne ? Bons et mauvais rituels chez Liudprand de Crémone », p. 19-61, particulièrement p. 54-56. L'auteur parle p. 55 d' « amnésie systématique » dans les textes de HROTSVITHA DE GANDERSHEIM (Gesta Ottonis, dans Hrotsvithae opera, III, 1, MGH, SS rer. Germ. in usum scholarum separatim editi, Berlin, 1902, éd. 1965, P. WINTERFELD éd., p. 201-228), M. GOULET trad., Hrotsvitha de Gandersheim, œeuvres poétiques, Grenoble, 2000, p. 161-204 et de LIUTPRAND DE CRÉMONE (Antapodosis, dans Liutprandi episcopi Cremonensis opera omnia, J. BECKER éd., MGH, SS. rer. Germ. in usum scholarum, Hanovre-Leipzig, 3e éd. 1915, p. 1-158), qui « oublient » de mentionner plusieurs révoltes au sein de la famille ottonienne et dont il sera question plus loin (seconde révolte d'Henri en 941, révoltes de Thankmar en 936 et de Liudolf en 953).

17..RUOTGER, c. 42, p. 271 ; WIDUKIND DE CORVEY, Renun gestarum Saxonicarum libri tres, H. E. LOHMANN et P. HIRSCH éd., MGH, SS rer. Germ. in usum scholarum separatim editi, Hanovre, 1935, 5e éd. 1977, p. 1-154, ici II, 39, p. 99 l'appelle aussi soror regis.

18..ADALBERT, Continuatio Reginonis anni 907-967, dans Reginonis abbatis Prumensis Chronicon cum continuatione Treverensi, F. KURZE éd., M.G.H., S.S. rer. Germ. in usum scholarum separatim editi, Hanovre, 1890, éd. 1989, p. 154-179, a. 929, p. 158.

19..IOCUNDUS, Translatio sancti Servatii, R. KOEPKE éd., M.G.H., S.S. XII, p. 85-126, ici c. 78, p. 123. A. DIERKENS et M. MARGUE « Memoria ou damnatio memoriae ? L'image de Gislebert, duc de Lotharingie », dans Retour aux 
sources : textes, études et documents d'histoire médiévale offerts à Michel Parisse, Paris, 2003, p. 869-890, particulièrement p. 884-890.

20..Sur les veuves au haut Moyen Âge, E. SANTINELLI, Des Femmes éplorées? Les veuves dans la société aristocratique du haut Moyen Âge, Villeneuve d'Ascq, 2003, qui montre p. 137-140 que certaines veuves, surtout à partir du $\mathrm{X}^{\mathrm{e}}$ siècle, pouvaient jouir d'une certaine liberté et parfois même décider de leur avenir en toute indépendance. Pour l'époque ottonienne, voir plus spécifiquement P. CORBET, « Pro anima senioris sui. La pastorale ottonienne du veuvage », dans M. PARISSE éd., Veuves et veuvage dans le Haut Moyen Âge, Paris, 1993, p. 233-253.

21..RICHER, Historiarum libri quatuor, Histoire de France, R. LATOUCHE éd. et trad., 2 vol., Paris, Les Classiques de L'Histoire de France au Moyen Âge, 1930-1937, tome 1, II, 19, p. 156-157 ; WIDUKIND, II, 26, p. 89 ; ADALBERT, a. 939, p. 161. Pour Louis, Gerberge présente de nombreux avantages : elle appartient à une famille royale (pratiques homogamiques), elle n'est pas directement apparentée aux Carolingiens, elle est la belle-sœur d'Hugues le Grand, et elle a des droits sur la Lotharingie.

22..Voir R. LE JAN, « Entre Carolingiens et Ottoniens, les voyages de la reine Gerberge », dans Les Assises du pouvoir, études offertes à Jean Devisse, Paris, 1994, p. 163-173, ici p. 168-169, repris dans EAD., Femmes, pouvoir et société dans le Haut Moyen Âge, Paris, 2001, p. 30-38 sous le titre « La Reine Gerberge, entre Carolingiens et Ottoniens », et K. LEYSER, Rule and Conflict in an Early Medieval Society : Ottonian Saxony, Londres, 1979, p. 53 : par ce mariage, Louis IV cherche à élargir ses bases en Lotharingie pour préparer son retour à la Francie occidentale.

23..Elle a alors environ 25 ans, et la tutelle sur son jeune fils Henri représente en réalité la mainmise sur le duché. Pour le roi, son remariage est donc potentiellement dangereux, et il cherche à unir sa sœur à un de ses fidèles, le duc de Bavière Berthold, qui choisit finalement d'attendre la majorité d'Hadwige, fille de Gerberge et de Gislebert : LIUTPRAND, IV, 31, p. 126.

24..Même type de situation entre Otton III et sa soeur Mathilde lors du mariage de celle-ci avec Ezzo, comte palatin : voir THIETMAR, IV, 60, p. 200. Ici, la situation semble même compliquée par le fait que l'union est de type hypogamique pour la princesse, dont le statut est donc ainsi dégradé par ce qui a dû être perçu par certains, dont Thietmar, comme une mésalliance, d'autant que les deux autres sœurs d'Otton III, vouées au cloître, s'engagent dans une carrière beaucoup plus prestigieuse à la tête des abbayes royales de Gandersheim et Quedlinburg. Mais Otton III réagit de façon pragmatique, et l'affaire n'engendre pas de conflit entre le frère et sa sœur.

25..WIDUKIND, I, 31, p. 44.

26..RICHER, II, 86, p. 274-275 sur la fête de Pâques 949 à Aix-la-Chapelle. R. LE JAN, « La reine Gerberge », loc. cit., p. 30-31.

27..D. LETT, Histoire des frères et sœurs, op. cit., p. 10.

28..Description par THIETMAR, VI, 3, p. 276.

29..Ibid., VI, 35, p. 316-318, 41, p. 324, 51, p. 338, 67, p. 356 et 87, p. 378

30..Ibid., VII, 54, p. 466 et VII, 66, p. 480.

31..Ibid., VIII, 18, p. 516. WIPON, Gesta Chuonradi imperatoris, dans Wiponis Opera, H. BRESSLAU éd., MGH, SS rer. Germ. in usum scholarum separati editi, Hanovre-Leipzig, 1915, éd. 1977, p. 3-62, c. 1, p. 9 montre Cunégonde, devenue veuve en 1024, s'appuyant sur ses frères pour gouverner jusqu'à la prochaine élection royale.

32..Synthèse sur le mariage à l'époque ottonienne par P. CORBET, « Le Mariage en Germanie ottonienne d'après Thietmar de Mersebourg ", dans M. ROUCHE et J. HEUCLIN éd., La Femme au Moyen Âge, Maubeuge, 1990, p. 187-212, particulièrement p. 199-207.

33..Veuve, elle avait pris le voile, avant d'épouser Henri, d'où l'opposition à leur union menée par l'évêque Sigismond de Halberstadt : THIETMAR, I, 5-6, p. 8-10. 
34..Ibid., I, 9, p. 14 : naissance de Thankmar, amour déclinant d'Henri pour Hatheburge et finalement mariage avec Mathilde.

35..WIDUKIND, II, 9, p. 73 et 11, p. 76-77 ; THIETMAR, II, 2, p. 40.

36..Indications éclairantes de R. SCHÖLKOPF, Die sächsischen Grafen, op. cit., p. 35-36.

37..Il a en effet déposé sur l'autel de l'église ses armes et son torque d'or, symboles de sa qualité de guerrier et de prince : WIDUKIND, II, 11, p. 76-77.

38..Sur ce mariage, HROTSVITHA, Gesta Ottonis, v. 66-124, p. 206-208 ; K. LEYSER, « The Ottonians and Wessex », version anglaise de "Die Ottonen und Wessex ", Frühmittelalterliche Studien, 17, 1983, p. 73-97 repris dans Communications and Power in Medieval Europe - the Carolingian and Ottonian Centuries, T. REUTER (éd.), Londres-Rio Grande, 1994, p. 73-104.

39..WIDUKIND, III, 10, p. 110.

40..L'oncle paternel, le mauvais oncle comme très souvent quand il est laïque ! Thietmar n'écrit-il pas « les oncles se déchaînent toujours contre les fils de leurs frères » (VI, 53, p. 340 : « semper patrui in fratrum filios seviunt ») ? L'opposition neveu/oncle paternel se manifeste en effet très fréquemment dans les sources, pour des raisons de concurrence pour l'influence auprès du père et frère, comme c'est le cas pour Liudolf et Henri, ou pour des questions d'héritage. Le patruus cherche alors à profiter de la faiblesse de l'orphelin pour rattraper le « manque à gagner » de l'héritage paternel en récupérant la part du frère décédé.

41..HROTSVITHA, v. 154-155, p. 209.

42..Ibid., v. 25-32, p. 205. On peut noter qu'elle ne fait ici pas allusion à l'existence de leur demi-frère Thankmar. Sur ce passage, J. L. NELSON, « Perception du pouvoir chez les historiennes du Haut Moyen Âge », dans M. ROUCHE et J. HEUCLIN éd., La Femme au Moyen Âge, op. cit., p. 75-83, particulièrement p. 78-79.

43..Les premiers nés d'Henri I ${ }^{\text {er }}$ (Thankmar) et d'Otton $\mathrm{I}^{\text {er }}$ (Guillaume et surtout Liudolf) sont écartés, soit en raison de leur naissance « irrégulière », soit pour révolte.

44..WIDUKIND, I, 41, p. 60.

45..Ibid., II, 12, p. 78 : nimia regnandi cupiditate illectus ; II, 15, p. 79 : ardens cupiditate regnandi.

46..Vita Mathildis reginae posterior, GH. Pertz éd., MGH, SS. IV, Hanovre, 1841, éd. 1968, p. 283-302, c. 6, p. 287 et c. 9, p. 289 (il existe une édition plus récente : Die Lebensbeschröburger der Kônigir Mathilde, B. SCHÜTTE éd., MGH, SS rer. Germ. LXVI, 1994) et THIETMAR, I, 21, p. 28. Selon Karl Leyser, « The Ottonians and Wessex », loc. cit., p. 86 et 88, ce soutien à Henri provient surtout du mariage prestigieux d'Otton avec Edith, princesse royale de Wessex. Face à sa belle-fille, la reine-mère n'a plus qu'une influence limitée, elle passe au second plan alors qu'elle peut encore tout à fait contrôler le jeune Henri encore célibataire.

47..Vita Mathildis reginae post., c. 9, p. 289. M. LINTZEL, « Heinricus natus in aula regali », dans Miszellen zur Geschichte des zehnten Jahrhunderts, Berlin, 1953, repris dans ID., Ausgewählte Schriften. Band II.: Zur Karolinger - und Ottonenzeit, zum hohen und späten Mittelalter, zur Literaturgeschichte, Berlin, 1961, p. 276-282.

48..K. LEYSER, « The Ottonians and Wessex », loc. cit., p. 87.

49..WIDUKIND, II, 2, p. 67.

50..Il est né sans doute entre 919 et 922.

51..WIDUKIND, II, 12, p. 78 (938) et Ibid., II, 15, p. 79 (939).

52..Ibid., II, 29, p. 91.

53..Ibid., II, 31, p. 92-93 ; HROTSVITHA, v. 316-335, p. 213-214 (pour la poétesse, Henri refuse de se joindre à cette tentative d'assassinat) et THIETMAR, II, 21, p. 62.

54..WIDUKIND, II, 36, p. 95 ; HROTSVITHA, v. 156-159, p. 209.

55..G. ALTHOFF, Spielregeln der Politik im Mittelalter. Kommunikation in Frieden und Fehde, Darmstadt, 1997.

56..J.-M. MOEGLIN, « Pénitence publique et amende honorable au Moyen Âge », Revue Historique, 604, octobre-décembre 1997, p. 225-269 (en particulier p. 225 et 233). 
57..HROTSVITHA, v. 336-371, p. 214-215. Nombreux éléments communs dans la réconciliation d'Henri II avec ses beaux-frères : THIETMAR, VII, 9, p. 408.

58..HROTSVITHA, v. 376-377, p. 215.

59..Étude approfondie dans L. LELEU, Les Fêtes ottoniennes (919-1024), Mémoire de Maîtrise sous la direction de R. LE JAN, Université Charles-de-Gaulle - Lille III, 2000, particulièrement p. 29-36 et p.143-151.

60..Sur l'importance de la notion de crise dans l'historiographie ottonienne, L. BORNSCHEUER, Miseriae regum. Untersuchungen zum Krisen- und Todesgedanken in den herrschaftstheologischen Vorstellungen der ottonischsalischen Zeit, Berlin, 1968, (Arbeiten zur Frühmittelalterforschung 4), p. 16 sq.

61..WIDUKIND, II, 4, p. 70.

62..K. LEYSER, Rule and Conflict, op. cit., chapitre 1 « The Roots of Discord », p. 9-22 et G. ALTHOFF, Adels und Königsfamilien im Spiegel ihrer Memorialüberlieferung. Studien zum Totengedenken der Billunger und Ottonen, Munich, (Münstersche Mittelalter-Schriften 47), 1984.

63..À celui de Lunebourg répond celui de Kemnade, fondé sur l'héritage de Wicmann, qui fait de sa fille Frederun l'abbesse. Voir N. WARLOP, Les Fondations de monastères féminins saxons du VIII au début du XIe siècle, Mémoire de Maîtrise sous la direction de R. LE JAN, Université Charles-de-Gaulle - Lille III, 2001, p. 106.

64..WIDUKIND, II, 11, p. 75-76.

65..THIETMAR, II, 12, p. 50.

66..Ibid., II, 6, p. 44-46 : ils participent à la révolte de Liudolf.

67..Ibid., II, 31, p. 76-78.

68..G. ALTHOFF, Adels - und Königsfamilien, op. cit., p. 119.

69..R. LE JAN, « La Vengeance d'Adèle ou la construction d'une légende noire », dans D. BARTHÉLEMY, F. BOUGARD et R. LE JAN dir., La Vengeance, 400-1200, Rome, Collection de l'Ecole française de Rome-357, 2006, p. 325-340.

70..F. W. OEDIGER, « Adelas Kampf um Elten (996-1002) », Annalen des historischen Vereins für den Niederrhein, 155-156, 1954, p. 67-86. Sur ce type de contestation, voir R. LE JAN, « Male ordine tenent. Transferts patrimoniaux et conflits dans le monde franc (VII ${ }^{-} \mathrm{X}^{\mathrm{e}}$ siècle) », dans EAD., Femmes, pouvoir et société dans le Haut Moyen Âge, op. cit., p. 132-148. Cette opposition d'Adèle suggère le fait qu'elle n'a pas dû être consultée ou que ces dons ont été faits contre sa volonté : il n'y a donc sans doute pas eu laudatio parentum au moment de l'établissement de l'acte accordant ces biens fonciers au monastère. Sur ce sujet, voir SD. WHITE, Custom, Kinship and Gifts to Saints, the laudatio parentum in Western France, 1050-1250, Chapell Hill-Londres, 1988.

71..ALPERT DE METZ, De diversitate temporum libri duo, G.H. PERTZ éd., M.G.H., S.S. IV, Hanovre, 1841, éd. 1968, p. 702-723, ici livre 1.

72..Ibid., I, 2-3, p. 702-703.

73..Ibid., I, 2, p. 702 : suo generi degeneri conubio inferre audebat.

74..Voir H. PLATELLE, «L'épouse 'gardienne de l'âme et de la vie de son mari' », dans M. ROUCHE et J. HEUCLIN éd., La Femme au Moyen Âge, op. cit., p. 171-184, ici p. 180-181.

75..Ibid., p. 183.

76..Sur cette situation particulière et peu confortable des cadets dans le royaume de France, G. DUBY, « Les " jeunes » dans la société aristocratique dans la France du Nord-Ouest au XII ${ }^{e}$ siècle », dans Annales ESC, 1964, p. 835-846, repris dans ID., Féodalité, Paris, 1996, p. 1383-1397. Comparaison des deux espaces par R. LE JAN, « De la France du Nord à l'Empire : réflexions sur les structures de parenté au tournant de l'an mil », loc. cit. 77..THIETMAR, I, 24, p. 30. Chez Liutprand, la rébellion d'Henri contre son aîné est aussi présentée comme d'inspiration diabolique : IV, 18-20, p. 114-115.

78..THIETMAR, II, 2 , p. 38. Sur la position de Thietmar au sujet des Slaves païens, G. BÜHRER-THIERRY, « Un évêque face aux païens : Thietmar de Mersebourg et les Liutizes », dans S. GOUGENHEIM dir., Retour aux sources, op. cit., p. 591-599. 
79..THIETMAR, IV , 57-58, p. 196-198 : quand le duc Boleslas de Pologne chasse ses frères à la mort de leur père, c'est « pour être le seul maître ».

\section{RÉSUMÉS}

Alors que les relations familiales sont largement idéalisées dans les sources narratives de l'époque ottonienne comme fondées sur l'entente, la solidarité et l'affection, de nombreux exemples témoignent néanmoins de rapports dans la réalité parfois tendus voire violents à l'intérieur même des fratries et surtout entre frères ou demi-frères, conflits plus ou moins graves et durables qui peuvent avoir des répercutions sur l'ensemble de la parenté. Ils s'expliquent en général par le sentiment d'injustice et de jalousie ressenti par un des membres de la fratrie qui considère ses intérêts comme lésés ou menacés, particulièrement à l'occasion d'un héritage ou de la revendication d'un honneur. La famille apparaît donc comme une réalité sociale complexe, comme le siège de compétitions et d'intérêts parfois divergents : c'est particulièrement le cas dans la famille royale ottonienne qui passe dans les années 930 à une nouvelle structure d'organisation, celle de la lignée, caractérisée notamment par l'instauration de la primogéniture et donc la relégation des cadets au second rang.

Brothers and Sisters at War in Tenth-Century Germany. Whereas narrative sources from the Ottonian period often idealize family relationships as based upon understanding, solidarity and love, there are at the same time numerous instances of real strained or even violent connections within the sib, for the most between brothers or half-brothers. These more or less serious and persistent conflicting situations may have consequences for the kinship as a whole. They originate mostly in the injustice and jealousy felt by a sib member who thinks his interests are impaired or threatened, specially when an inheritance or an honour is at stake. So family appears to be a complex social reality, the seat of competition and of interests that might differ: this is particularly the case in the Ottonian royal family which in the 930's becomes a lineage, through the adoption of primogeniture and so the younger brothers'relegation to the second rank.

\section{INDEX}

Mots-clés : aristocratie, conflit, genre, Germanie, Ottoniens, parenté, relations familiales

Keywords : aristocracy, conflict, family relationships, gender, Germany, kinship, Ottonians

\section{AUTEUR}

\section{LAURENCE LELEU}

Université Paris I-Panthéon-Sorbonne - LAMOP, UMR 8589, 1 rue Victor Cousin, 75005 Paris 